\title{
LA PLANIFICACIÓN LINGÜÍSTICA DESDE UNA SOCIOLOGÍA SISTÉMICA DEL LENGUAJE: UN ANÁLISIS DESDE LOS CASOS DEL MAPUDUNGÚN, EL SAMI Y EL MAORI ${ }^{1}$
}

\author{
LANGUAGE PLANNING FROM A SYSTEMIC SOCIOLOGY OF \\ LANGUAGE: AN ANALYSIS ON THE CASES OF MAPUDUNGÚN, \\ SAMI AND MAORÍ
}

\author{
CÉSAR CISTERNAS IRARRÁZABAL \\ Universidad de La Frontera \\ cesar.cisternas@ufrontera.cl
}

ARTURO VALLEJOS-ROMERO

Universidad de La Frontera

arturo.vallejos@ufrontera.cl

\section{RESUMEN}

En el presente trabajo se propone un acercamiento sociológico al problema de la planificación lingüística, planteando una perspectiva fundamentada en una lectura de los avances de la sociolingüística desde la teoría de sistemas sociales. El enfoque comprende la planificación lingüística como una estrategia de regulación del contacto lingüístico que opera en los sistemas de interacción -o sea, en la práctica comunicativa de los hablantes-. Este se aplica, a modo de ejemplo, a distintas aristas de la planificación del mapudungún, el sami y el maorí. Se concluye que la planificación se ejecuta mediante la introducción en el entorno de los sistemas de interacción de distinciones (hablar/no hablar, enseñar/no enseñar, etc.) que pretenden reorientar la operación de los sistemas. Se destaca la relevancia de la consideración de las condiciones sociohistóricas del contacto y la regulación en el análisis de estos procesos. Adicionalmente, se advierte que el análisis de los casos latinoamericanos debe tener en cuenta que los procesos de diferenciación funcional de estas sociedades tienen características particulares, lo que las lleva a estructurarse de un modo distinto a aquellas sociedades como las europeas.

Palabras clave: Planificación Lingüística, Sociología del Lenguaje, Teoría de Sistemas Sociales.

${ }^{1}$ Este artículo ha sido financiado por la Dirección de Investigación de la Universidad de La Frontera a través del proyecto DFP19-0030 y el proyecto Fondecyt Regular 1150576. 


\section{ABSTRACT}

The present article proposes a sociological approach to language planning, drawing a perspective based on an interpretation of the advances made by the sociolinguistics in the matter from the social systems theory. The theoretical framework comprehends language planning as a strategy to regulate language contact that operates in the interaction systems -that is, in the speakers' communicative practices-. This approach is applied, as example, to different aspects of the planning of Mapudungún, Sami, and Maorí. It is concluded that the planning is executed through the introduction of distinctions (to speak/not to speak, to teach/not to teach, etc.) in the environment of the interaction systems. The relevance of the consideration of the sociohistorical conditions of language contact and regulation in the analysis of these processes it is highlighted. In addition, a warning is made about the analysis of Latin-American cases, which must consider that these societies are characterized by a concentric functional differentiation, that make them differ from the societies of the central modernity.

Keywords: Language Planning, Sociology of Language, Social Systems Theory.

Recibido: 29/03/2019. Aceptado:10/10/2019.

\section{INTRODUCCIÓN}

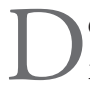
esde que el concepto de planificación lingüística fuese propuesto por Einar Haugen en 1959, se estructuró un campo de investigación que se ha ido consolidando en las décadas siguientes (Omdal, 2006). Sin embargo, todavía no se ha logrado construir una teoría general que permita abordar totalmente la complejidad del fenómeno (Amorós-Negre, 2017; Cooper, 1997; Ricento, 2006).

El presente trabajo plantea un nuevo marco teórico-conceptual que problematiza sociológicamente la planificación lingüística, construido a partir de una lectura de los avances de la sociolingüística desde la teoría de los sistemas sociales de Niklas Luhmann. La propuesta es empleada para analizar los casos del sami, el maorí y el mapudungún, como ejercicio para su aplicación empírica.

La planificación lingüística corresponde a un conjunto de acciones cuya finalidad es regular las funciones, aspectos formales (alfabeto, ortografía, gramática) y enseñanza de la lengua (Cooper, 1997; Hornberger, 2006). Si bien desde esta definición sociolingüística la lengua aparece como su objeto último, una aproximación sistémica al problema obliga a desechar este punto de vista. De acuerdo con Luhmann (1996) la sociedad es un sistema constituido por sistemas parciales de comunicación para los cuales los seres humanos o sistemas psíquicos constituyen el entorno. En este contexto, el lenguaje -y las lenguas como expresiones particularizadas del lenguaje- se presenta como un médium que posibilita el acoplamiento estructural de los sistemas psíquicos y sociales (Lu- 
hmann, 2006), vale decir, entre conciencia y comunicación.

La planificación lingüística busca regular el contacto lingüístico reduciendo o ampliando las posibilidades de selección de médiums que tienen los sistemas psíquicos y sociales para acoplarse. Tal como lo recalca Joseph (2015), dicho contacto tiene lugar en la interacción comunicativa de los hablantes, es decir, se materializa únicamente en su práctica lingüística. Así, desde una interpretación luhmanniana, la regulación que busca la planificación lingüística opera sobre los sistemas de interacción, pretendiendo reconducir la elección lingüística de los sistemas psíquicos al momento de involucrarse en el proceso de comunicación.

Tomando en consideración lo planteado, el presente trabajo se propone comprender la planificación lingüística como una estrategia de regulación desde la perspectiva sistémica. En este marco, se considera, junto a Mascareño (2011), que la intervención regulatoria actúa sobre el entorno de los sistemas (sujetos, instituciones) -nunca sobre estos directamente- y que será efectiva sólo en cuanto logre que los sistemas se vuelvan receptivos a las distinciones que desde el entorno se introducen mediante la intervención.

Las implicaciones de estas definiciones preliminares, que derivan en el desarrollo de la propuesta teórica, son contrastadas con los casos de planificación del mapudungún, el maorí y el sami. Estas iniciativas de planificación para la revitalización cuentan con una serie de puntos comunes, por vincularse todas a lenguas indígenas minorizadas, además de encontrarse ampliamente documentadas, lo cual facilita su análisis teórico.

El trabajo se desarrolla en tres secciones. En la primera, se expone una concepción sistémica de los contextos de contacto lingüístico. En la segunda se problematiza la planificación lingüística como una estrategia de regulación desde el marco de la orientación sistémica contextual. Mientras que en la tercera se analiza, desde la perspectiva teórica construida, las estrategias de regulación implementadas en los casos del mapudungún, el maorí y el sami. Por último, se plantean las conclusiones a las que ha conducido el trabajo.

\section{UNA APROXIMACIÓN SISTÉMICA AL CONTACTO LINGÜÍSTICO}

La planificación lingüística tiene lugar siempre en contextos en que confluyen dos o más lenguas o variantes lingüísticas. En consecuencia, la construcción de un marco teórico para comprender este fenómeno debe necesariamente problematizar el contacto lingüístico.

El contacto entre lenguas se puede producir por diversas razones: intercambio comercial, guerra, integración forzada de población foránea o inmigración de hablantes de otras lenguas; teniendo consecuencias tanto a nivel lingüístico como social. 
De acuerdo con la teoría de sistemas sociales de Luhmann (1996) la sociedad constituye un sistema de comunicación que está conformado por sistemas parciales, como política, derecho, economía, etc. Y, si bien estos sistemas sociales son sistemas de comunicación -lo que en un principio podría llevar a pensar que el individuo juega un papel importante en el proceso-, son autónomos y autopoiéticos. O sea, operan con independencia a partir de sus propias estructuras, siendo capaces de reproducirse a sí mismos sin intervención del entorno. En este esquema, entonces, el individuo como tal queda excluido, volviéndose visible sólo cuando entabla comunicación con otro u otros individuos.

Desde el punto de vista de la teoría de sistemas, la diferencia lingüística entre comunidades de habla diferentes se elude. Esto en cuanto los términos de interacción e intercambio entre sociedades quedan determinados por los medios de comunicación simbólicamente generalizados, que surgen de la diferenciación funcional producida y acentuada por la constante operación autorreferencial de los sistemas parciales (Luhmann, 1998). Tales interacciones dependerán de relaciones de poder, intercambios de dinero, etc., por lo que el entendimiento entre las sociedades será posible, aunque los individuos que conforman ambos grupos no manejen la lengua del otro grupo.

En consecuencia, para la teoría de sistemas el contacto lingüístico se vuelve problemático únicamente en lo que se denomina sistemas de interacción, es decir, en la comunicación concreta de dos o más individuos, o entre un individuo y una institución. Esto concuerda con la descripción del contacto planteada por Joseph (2015), de acuerdo con la cual el contacto de lenguas se produce en situaciones comunicativas concretas y no en un nivel general o abstracto de la sociedad.

Este ajuste entre la interpretación sociolingüística del contacto lingüístico y la concepción sistémica del individuo y sus competencias lingüísticas vuelve interesante abordar desde la teoría de los sistemas sociales los problemas relacionados con el contacto, tales como la planificación lingüística.

\section{LA PLANIFICACIÓN LINGÜÍSTICA COMO REGULACIÓN SISTÉ- MICA CONTEXTUAL}

La planificación lingüística refiere al conjunto de acciones deliberadas que se orientan a definir las funciones, forma (gramática, ortografía, alfabeto) y espacios de enseñanza de una lengua (Cooper, 1997). En una visión que pone un mayor acento en los hablantes, Deumert (2009) la describe como un conjunto de esfuerzos destinados a modificar las prácticas lingüísticas de una determinada comunidad de habla.

De acuerdo con Heller (2013), a menudo se realiza una aproximación a las cuestiones de política y planificación lingüística desde un marco asociado a la elec- 
ción racional. De tal modo, se cae en el error de representar el problema como si implementar un determinado conjunto de medidas condujera, automáticamente, a que cierto sector de la población adoptara las prácticas lingüísticas consideradas deseables por la iniciativa de planificación. A este respecto, la mirada sociolingüística recalca la relevancia de prestar atención al contexto social y simbólico en el que se emplean las lenguas y considerar la dirección del cambio social que tiene lugar en la sociedad que se desea investigar o intervenir (Deumert, 2009). En tal sentido, se debe tener en cuenta que la planificación lingüística está siempre imbricada con cuestiones relativas a la historia, la política y la inequidad (May, 2015).

Desde la sociología del lenguaje de Bourdieu $(1991 ; 1999)$ la planificación lingüística puede ser interpretada como una regulación de los mercados lingüísticos; esto es, de los espacios en los que confluyen los hablantes y se emplean las distintas lenguas en las cuales estos tienen competencia. Los sujetos que interactúan en estos mercados muchas veces no se encuentran en igualdad de condiciones, estableciéndose interacciones determinadas por relaciones de poder configuradas a partir del nivel de competencia que estos tienen en cada lengua que circula en los distintos mercados (Bourdieu, 1991). El aporte de esta perspectiva reside en poner énfasis en las condiciones sociohistóricas en las cuales tiene lugar la comunicación que establecen los hablantes.

Una lectura luhmanniana de la propuesta de Bourdieu $(1991 ; 1999)$ conduce a plantear que las condiciones en las cuales cada hablante se involucra en los sistemas de interacción no son equivalentes, estando influidas por el repertorio lingüístico con el que cuentan. Así, desde esta perspectiva, la planificación lingüística se orienta a regular las condiciones en que los sujetos entablan relaciones en los sistemas de interacción.

Tradicionalmente, como lo destaca Nahir (1998), la planificación lingüística fue concebida como una acción que podía ser emprendida únicamente por un grupo selecto de agentes que constaba con ciertas posiciones privilegiadas en lo que Bourdieu (1991) denomina campo lingüístico, es decir, el escenario social estructurado por relaciones de poder en el que se sitúan los hablantes. De tal modo, quedaba como una iniciativa restringida a agentes tales como academias de lenguas, gobiernos o comisiones ad hoc.

Desde un tiempo a esta parte, se ha difundido una visión más compleja sobre la materia, de acuerdo con la cual cualquier individuo u organización con posibilidad de influir sobre la lengua puede llevar a cabo iniciativas de planificación (Omdal, 2006). De tal manera, emergen como potenciales planificadores distintas instancias gubernamentales o altamente especializadas (academias de lenguas, grupos de expertos, etc.), en una orientación de arriba hacia abajo (top-down). Pero la propia comunidad de habla también puede adoptar este rol mediante proyectos locales implementados que buscan tener impacto en niveles territoriales mayores (bottom-up) (Grenoble y Whaley, 2005; Wright, 2004). A este respecto, Liddi- 
coat y Baldauf (2008) han sugerido que los contextos locales son fundamentales en estos procesos, ya que son los actores, en tal nivel, los destinatarios finales de este tipo de políticas y, por ende, quienes, a través del mantenimiento/reorientación de prácticas lingüísticas, determinarán su éxito o fracaso.

Ahora bien, en contextos bilingües -o multilingües- el poder se vuelve aún más relevante en las relaciones sociolingüísticas (véase Fishman, 1995). Por lo tanto, el reconocimiento/no reconocimiento de una lengua y la promoción de su ingreso a los mercados formales y prestigiosos dista de ser un debate meramente técnico, en cuanto constituye un asunto político, que puede generar y/o acentuar conflictos en lugar de resolverlos (Wiley, 1996).

Por lo tanto, la propuesta teórica de este trabajo, que pretende abordar la regulación del contacto lingüístico, se basa en el modelo de orientación sistémica contextual. La planificación lingüística, tal como se representa en la Figura 1, es un fenómeno multinivel en el que se encuentran imbricadas una serie de instancias, por tanto, abarca un gran número de coordinaciones, cooperaciones y conflictos actuales y potenciales. De acuerdo a Mascareño (2011) es precisamente en estas instancias de coordinación de los distintos sistemas que comparten el entorno, donde existe la posibilidad de transformación de la sociedad mediante la intervención.

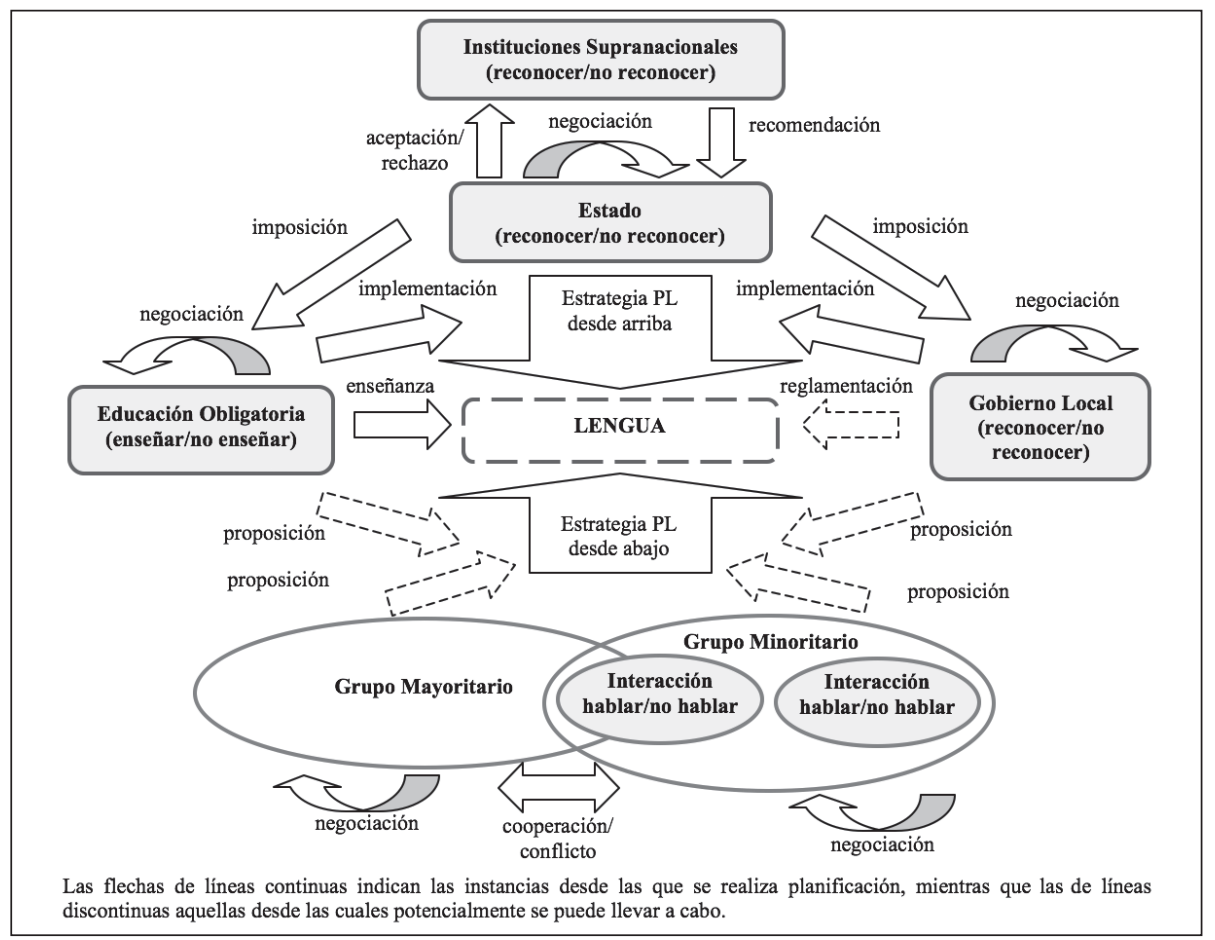

Figura 1. Actores y Dinámicas Involucrados en la Planificación Lingüística en Contextos Bilingües. Fuente: Elaboración propia. 
Wilke (2006) recalca que, en el contexto de una modernidad caracterizada por un alto grado de diferenciación funcional, las intervenciones que buscan la reorientación de las operaciones de un determinado sistema deben procurar conservar la autonomía de aquel, evitando la desdiferenciación o la imposición del primado de un sistema social sobre otros. Partiendo de esta premisa, y teniendo en cuenta que la intransparencia de los sistemas ${ }^{2}$ dificulta el proceso de reorientación de estos, Mascareño (2011) arguye que el desafío de la regulación se encuentra en introducir distinciones que constituyan alternativas de selección dotadas de sentido para el sistema a regular. Así, según este autor, "la orientación contextual no controla, coordina, y coordina operaciones clausuradas de sistemas autopoiéticos intransparentes entre sí a través de una sincronización de operaciones en el momento y en el modo en que tales sistemas realizan esas operaciones" (Mascareño, 2011: 7).

Entonces, tal como lo indica Vallejos (2008), lo que persigue este tipo de intervención es hacer que el sistema a intervenir perciba el cambio como imprescindible para continuar su operación. Esto implica que, en el caso de la planificación lingüística, se debe lograr que en los sistemas de interacción la posibilidad de seleccionar un médium de acoplamiento entre sistemas psíquicos y sociales (lengua) diferente al dominante aparezca como válida. Esto resulta consistente con la aproximación sociolingüística, según la cual el desafío último de la planificación lingüística no es brindarle un mayor estatus legal a la lengua o introducirla en la escuela, sino lograr que las familias hablen la lengua en peligro y se la enseñen a los niños (Romaine, 2002).

Desde la década de 1970, con el surgimiento de los primeros movimientos indígenas y de minorías étnicas en distintos países, se comienza a consolidar una semántica del reconocimiento a nivel internacional, sustentada en gran medida en las directrices normativas adoptadas por instituciones supranacionales. De este modo, los ulteriores conflictos étnicos se encuentran en un mejor pie para que su demanda por reconocimiento sea atendida. Y es que tal como lo señala Wright (2004) el nuevo contexto global, caracterizado por cierto grado de integración política y normativa a nivel supranacional, impide a los Estados nacionales ejercer el control hegemónico y homogeneizador que los caracterizó en el siglo XIX. Así, estos se ven presionados a reorientar sus dinámicas comunicacionales respecto a la definición del poder/no poder en la relación con las minorías étnicas, aunque siguen siendo el actor con más poder en un territorio nacional a la hora de definir la política lingüística (Romaine, 2002).

En este sentido, la semántica del reconocimiento condiciona de algún modo las comunicaciones de los sistemas políticos nacionales, que empiezan a contem-

${ }^{2}$ Dentro de la teoría de sistemas sociales, el fenómeno de la intransparencia refiere al hecho de que los lenguajes sistémicos son ilegibles entre sí. 
plar a las minorías como entidades políticas diferenciadas de la sociedad nacional mayoritaria. Para comprender adecuadamente el modo en que se da esta reorientación es preciso considerar que el Estado no es un cuerpo monolítico, sino que se compone de una multiplicidad de entidades distribuidas en distintos niveles geográficos, existiendo la posibilidad de conflicto entre estas diversas instancias (Gupta, 1995; Nugent, 2004). Por ende, lo más probable es que la semántica del reconocimiento irrite de formas disimiles a las distintas estructuras del Estado.

A nivel lingüístico, el cambio en las selecciones de las polaridades poder/no poder con respecto a las minorías étnicas, influye en la apreciación del capital lingüístico minoritario. Se genera, en consecuencia, un impacto en el acceso de tal lengua a los mercados formales -o funciones lingüísticas poderosas en el lenguaje de Fishman (2001)-, vale decir, comunicación gubernamental, trabajo, educación, medios de comunicación, entre otros.

La planificación lingüística, concebida como regulación por orientación sistémica contextual, busca introducir la distinción hablar $\mathrm{x} /$ no hablar $\mathrm{x}^{3}$ en el entorno de los sistemas de interacción y guiarlos hacia la selección del primer lado de aquel. Esto es una premisa fundamental, ya que la regulación no se dirige a controlar la operación del sistema, pues es imposible, sino que pretende reorientar las selecciones que este realiza poniendo a su disposición otras alternativas relevantes. A lo que apunta una regulación es a la transformación de los entornos de los sistemas de interacción. Por ejemplo, una planificación relativa a las funciones de una lengua busca transitar desde entornos monolingües, en los que la única posibilidad de selección es hablar/no hablar la lengua dominante ${ }^{4}$, a entornos bilingües o multilingües, en los cuales se amplían las alternativas de selección para los sistemas de interacción.

En los procesos de planificación lingüística intervienen una serie de sistemas: político, educativo, científico, etc., y tienen lugar a una serie de conflictos y espacios de negociación. Una manera frecuente de procesar la complejidad que representan las nuevas dinámicas comunicacionales generadas por el conflicto es recurrir a las prestaciones del sistema jurídico. $\mathrm{Y}$ es que este sistema suele estar involucrado, mediante el acoplamiento estructural con otros sistemas, en la canalización de la resolución de conflictos, debido a su capacidad de cristalizar ciertas semánticas a través de nuevas estructuras de comunicación (Luhmann, 1996),

\footnotetext{
${ }^{3}$ Esta distinción aplica en el caso de la planificación de las funciones que ocupa una lengua; otros ámbitos de planificación tendrán sus propias distinciones. Así, en el plano de la adquisición estas se relacionarán con enseñar/no enseñar la lengua y aprender/no aprender la lengua; mientras que en lo relativo a la regulación de los aspectos formales el abanico de distinciones podrá incluir escribir con $\mathrm{x} /$ no escribir con $\mathrm{x}$, estandarizar/no estandarizar, usar/no usar variedad estándar, etc.

${ }^{4}$ En este tipo de escenarios, la selección del segundo lado implicaría no establecer comunicación verbal.
} 
La legislación aparece, así, como un camino para dar estabilidad temporal a las estructuras comunicacionales resultantes de la negociación entre los distintos actores involucrados en el conflicto por la política lingüística.

\section{TRES CASOS DE PLANIFICACIÓN DE LENGUAS INDÍGENAS: MA- PUDUNGÚN, MAORÍ Y SAMI}

Esta sección se orienta a aplicar el enfoque expuesto previamente a tres casos empíricos: el mapudungún en Chile, el maorí en Nueva Zelanda y el sami en Noruega. Los procesos que han atravesado estos idiomas mantienen una serie de puntos en común, siendo todos ellos lenguas indígenas minorizadas y que han perdido vitalidad producto de prolongados periodos de colonialismo. A esto se suma la conformación de movimientos político-identitarios que abogan por su reconocimiento, los que han derivado, en mayor o menor medida, en acciones emprendidas por los respectivos Estados orientadas a su protección y promoción.

Debido a las limitaciones de espacio, el trabajo se centra en analizar una de las aristas de la planificación lingüística en cada caso. De tal modo, se aborda la planificación funcional del sami, la formal del mapudungún, y la de adquisición del maorí.

\subsection{La Planificación Funcional del Sami en Noruega}

El Estado noruego respetó, durante las primeras décadas tras su fundación en 1814, la opción de que los samis usaran y transmitieran su lengua. Sin embargo, tras las protestas de sectores de la clase alta y el surgimiento de ánimos nacionalistas y civilizatorios a mediados del siglo XIX, la disposición hacia la lengua indígena cambió radicalmente. Se inició, de esta manera, una campaña de asimilación, entre cuyas estrategias se encontraba la instrucción obligatoria en noruego (Minde, 2005). Con el tiempo el sami se prohibió en las escuelas y fue crecientemente estigmatizado a nivel de la sociedad general (Beach, 1994). Hablar noruego se transformó, así, en la única selección posible para los sistemas de interacción en los mercados lingüísticos formales, puesto que en su entorno sólo se encontraba disponible la distinción hablar noruego/no hablar noruego.

El escenario que enfrentaba el sami se mantuvo inalterado por décadas, fortaleciéndose su tendencia al desplazamiento. No obstante, hacia 1960, se conforma un movimiento político sami que reconfigura el panorama. Tal como lo sugiere Luhmann (1996), el conflicto origina nuevas dinámicas comunicacionales, en la forma de subsistemas que emergen en el seno de los sistemas sociales. En el caso 
noruego, la movilización sami, que en su variante más radical exigía el derecho a representación política mediante instancias autónomas, generó un clima de agitación que obligó a los partidos políticos a negociar una salida a la crisis hacia el final de la década de 1980 (Falch, Selle y Strømsnes, 2016). Logró, de tal forma, instalar la semántica del reconocimiento en la sociedad nacional, orientando la comunicación en este sentido, incluso antes de que los organismos supranacionales fijaran estructuras jurídicas (convenios, tratados) que la promovieran.

Se introdujeron distinciones en los entornos de los sistemas que incidieron en una reorientación comunicacional que tuvo lugar en múltiples planos. En el sistema político se estipuló una forma de autonomía no territorial basada en la creación de una institución legislativa propia (parlamento) y en el educativo se crea un sistema escolar con currículo propio para los samis, cuya administración es transferida al Parlamento Sami el año 2000 (Todal, 2003). Lo anterior se da en acoplamiento con el sistema judicial que otorga legalidad a la nueva configuración a través de leyes ad hoc y la Constitución.

La legislación noruega asegura, mediante leyes especiales, la posibilidad de emplear el sami en las cortes, de acceder en aquella lengua al ámbito religioso, de recibir respuesta en los servicios públicos y de ser atendido en las municipalidades de las zonas sami (Goverment of Norway, 2007), además del derecho a recibir educación primaria y los primeros años de secundaria en la lengua (Goverment of Norway, 2014). Todo en el marco de un compromiso de rango constitucional con la protección y promoción de una lengua que goza de estatus de co-oficialidad en el reino (Stortinget, 2014).

En su conjunto, estas acciones comprenden una estrategia de planificación lingüística desde arriba, que asigna a la lengua minoritaria las funciones de co-oficialidad, educación y religiosa. En todas estas esferas, el Estado noruego (sistema político), valiéndose de la legislación, introduce estructuras comunicacionales que otorgan poder a la lengua. Por ende, pone a disposición de los sistemas de interacción, que emergen en los ámbitos regulados (cortes, escuelas, gobiernos locales), la distinción hablar sami/no hablar sami como una alternativa dotada de sentido.

Además de esta protección desde arriba, en la propia comunidad de hablantes se han materializado iniciativas que buscan, simultáneamente, reforzar las dinámicas comunicacionales que pretenden orientar a los sistemas de interacción a que seleccionen el lado de la distinción correspondiente a hablar sami, y abrir espacios de uso para la lengua. Entre estas destacan la proliferación de la producción artística en sami, particularmente poesía (Gaski, 2011) y música (Diamond, 2007), además del desarrollo de medios de comunicación como radios, periódicos y un noticiario diario en televisión que incluye subtítulos en la lengua. La utilización de la lengua en internet también ha cobrado fuerza, principalmente como vía alternativa ante la falta de recursos para la inserción en medios más costosos 
como la televisión y los diarios (Pietikäinen, 2008). Asimismo, cabe mencionar la existencia de la Sámi allaskuvla (Universidad Sami), fundada en 1989 por el Ministerio de Educación noruego, que ha implantado una política lingüística que instaura el sami como lengua principal, constituyendo la lengua de instrucción y de administración (Bull, 2012).

Lo anterior ha llevado a que, actualmente, de los 60.000 samis que habitan en territorio noruego, unos 25.000 hablen la lengua tradicional (Ethnologue, 2018a), muchos de los cuales corresponden a nuevos hablantes que han adquirido la lengua en el contexto escolar (Aikio-Puoskari, 2018). Esto da cuenta de la eficacia de la experiencia de planificación lingüística del sami en el país, que ha logrado orientar exitosamente los sistemas de interacción hacia el hablar la lengua. Se ha contenido así, el grave proceso de desplazamiento que enfrentó la lengua desde inicios del siglo XX. En efecto, esta se ha expandido desde el ámbito exclusivamente familiar al que se vio confinada en la fase más acentuada de desplazamiento, hacia diversas funciones como la administración pública, educación, medios de comunicación, música y literatura (Aikio-Puoskari, 2018; Markelin y Husband, 2013; Todal, 2003).

\subsection{La Planificación de la Adquisición del Maorí}

En 1840, los colonizadores británicos que habían arribado a fines del siglo XVIII a Nueva Zelanda, firman el Tratado de Waitangi, a través del cual se comprometían a proteger a los maoríes, sus tierras y recursos (Barber, 2008). No obstante, el pacto no fue respetado por los europeos que, como consecuencia de las enfermedades y la llegada de las armas de fuego, sobrepasaron rápidamente en número a los nativos.

Hacia el final de la década de 1840, las ideas asimilacionistas predominaban en la elite. De este modo, entre los objetivos fundamentales del sistema nacional de educación neozelandés, instaurado hacia 1860, se encontraba la enseñanza del inglés a toda la población, apareciendo el maorí como un obstáculo para la adquisición de la lengua dominante (May, 1998). Así, el sistema educacional que se consolidaba operaba restringido a la distinción hablar inglés/no hablar inglés, prohibiéndose la lengua maorí en los establecimientos, lo que condujo a su rápido retroceso en cuestión de decenios (May y Hill, 2018).

Desde entonces, las políticas asimilacionistas del Estado neozelandés se suceden ininterrumpidas por un siglo, reforzándose constantemente una comunicación en que no se reconocía poder a los maoríes como entidad política diferenciada de la mayoría de origen europeo. Esto traería sus consecuencias: a mediados del siglo XX, gran parte de la población maorí había abandonado la lengua tradicional 
como medio de socialización intrafamiliar (Reedy, 2000). Ante tal escenario era claro el peligro que enfrentaba la lengua, pronosticándose, en aquel entonces, que dentro de 25 años desaparecería (Mahrooqi y Asante, 2012).

El descontento por la situación crítica de la lengua y la asimilación sistemática de la que son blanco los maoríes desemboca en la conformación de un movimiento maorí que demandaba al Estado cumplir con los compromisos contraídos a través del Tratado de Waitangi (Higgins y Rewi, 2015). Simultáneamente, comienza a tomar forma una serie de iniciativas desde abajo de planificación de la adquisición del maorí. Los Kohanga Reo o nidos lingüísticos, instancias de educación preescolar no formal gestionadas por las comunidades maoríes, resultan claves en la recuperación de la vitalidad de la lengua (May y Hill, 2018; Reedy, 2000). Esto en cuanto disputan el monopolio comunicacional del sistema educativo nacional, bajo el cual aprender maorí aparece como una alternativa carente de sentido para los sistemas de interacción.

Durante el transcurso de las décadas de 1970 y 1980 el Estado neozelandés cede ante las presiones del movimiento maorí, tomando medidas orientadas a su reconocimiento como pueblo y a la conservación de su cultura y su lengua (Mahrooqi y Asante, 2012). Estas iniciativas, tales como la oficialización de la lengua, la subvención a los nidos lingüísticos y el establecimiento de un sistema de educación bicultural, son plasmadas en una nueva legislación (Ley de Lengua Maorí, Ley de Educación), que -al igual que en el caso sami- encarna el catalizador del conflicto sociopolítico entre los maoríes y el Estado de Nueva Zelanda. Se introduce, de aquel modo, mediante la legislación, la distinción enseñar/no enseñar maorí en el sistema educacional neozelandés, al tiempo que el aprender maorí se presenta como alternativa dotada de sentido para los sistemas de interacción.

Actualmente, existen instituciones que ofrecen educación primaria y secundaria exclusivamente en maorí, y otras que cuentan con instancias de formación bilingüe, habiendo también instituciones de educación superior (Wānanga) que entregan al estudiante un entorno de aprendizaje maorí (Reedy, 2000). El alto número de estudiantes maoríes matriculados en modalidades de educación con instrucción exclusiva o mayoritaria en la lengua tradicional (New Zealand Ministry of Education, 2016; citado en May y Hill, 2018), da cuenta de la exitosa introducción de la distinción aprender maorí/no aprender maorí en los sistemas de interacción. Y, aunque persiste el desafío de generalizar el empleo de lengua tradicional en el ámbito familiar (May y Hill, 2018), la política lingüística neozelandesa ha logrado frutos notables si se considera que hoy en día unas 158.000 personas hablan la lengua, de un total de 600.000 maoríes que viven en el país (Ethnologue, 2018b). En este sentido, las diversas iniciativas de planificación, tanto desde arriba como desde abajo, han logrado introducir como contingencia la distinción hablar maorí/no hablar maorí en el entorno de los sistemas de interacción, y hacerla aparecer como alternativa dotada de sentido. 


\subsection{La Planificación Formal del Mapudungún en Chile}

Las primeras propuestas de planificación formal del mapudungún datan de la época de la colonia española. Aunque los mapuches se mantuvieron independientes del Imperio Español, se emprendieron varias misiones evangelizadoras en su territorio. En una sociedad con escasos niveles de diferenciación, el sistema político y el religioso se encontraban imbricados. Así, la Iglesia, a cargo de estas misiones, constituye un instrumento civilizatorio -y, por tanto, político- del Imperio. Es en este contexto, y con el fin de facilitar las tareas de conversión, que algunos misioneros documentan la lengua mapuche y confeccionan sistemas para su escritura. Entre los más difundidos en la época se pueden mencionar aquellos de Luis de Valdivia (1606), Andrés Febres (1765) y Bernardo Havestadt (1777) (Crow, 2015).

Tras la independencia chilena, en 1818, tiene lugar un proceso de construcción nacional, en cuanto, al igual que en las otras colonias emancipadas del continente, no se había conformado una identidad nacional consolidada (König, 2005). A pesar de que la naciente identidad chilena reivindicaba un vínculo con la resistencia mapuche a la corona española, nunca estuvo en entredicho que, en su dimensión lingüística, esta se hallaba fundada en el habla del castellano (Rojas, 2013). Tampoco existió una iniciativa concertada para asimilar lingüísticamente a los mapuches, quienes conservaban, de facto, su autonomía. El mapudungún, entonces, adquiere un carácter de no problema para la elite nacional, quedando invisibilizado (Rojas, 2013).

El panorama político cambia radicalmente hacia la segunda mitad del siglo XIX. La profunda crisis económica de 1857, gatillada por una confluencia de turbulencias internacionales y factores internos, lleva a pensar que la expansión de la producción agrícola hacia las tierras del sur es la solución a las vicisitudes que enfrentaba el país (Pinto, 2003). De este modo, el sistema económico, orientado por el código producir/no producir, apela a la necesidad de disponer de más tierra cultivable para dar estabilidad financiera al país. Al mismo tiempo, establece un acoplamiento con el sistema político, que procesa la comunicación de este como el deber de integrar y civilizar a los indígenas y extender sobre sus territorios la soberanía nacional. Se produce así una invasión militar al territorio mapuche que terminará con la incorporación de aquel pueblo a la sociedad nacional. El creciente contacto entre chilenos y mapuches, a través de la educación y el comercio, sumado a la estigmatización de la lengua indígena en la sociedad chilena, lleva a la consolidación del bilingüismo y el inicio del desplazamiento del mapudungún (Durán y Ramos, 1987; Lagos y Espinoza, 2013).

En este marco, entre los intelectuales y misioneros despierta un renovado inte-

${ }^{5}$ En este apartado se abordará sólo el ámbito de la planificación formal del mapudungún relativo a su representación gráfica, en cuanto es su arista más documentada. 
rés en documentar una lengua que se percibía en vías de extinción (Crow, 2015). Surgen así nuevas gramáticas y propuestas de escritura, como las de Lenz (18951897), Augusta (1903) y Moesbach (1962). De estas destaca particularmente la de Augusta, cuyo grafemario fue ampliamente utilizado durante las décadas siguientes (Zúñiga, 2001), convirtiéndose en el sistema de escritura con mayor difusión hasta entrada la segunda mitad del siglo XX.

La estructura premoderna de la sociedad chilena comienza a mutar con el avance del siglo XX, transitando hacia una creciente diferenciación funcional. Sin embargo, la diferenciación en las sociedades latinoamericanas no resulta policéntrica como aquella propia de los centros de la modernidad y que conduce a sistemas autónomos y autorreferenciales. Por el contrario, emerge una estructura concéntrica en la que "las distinciones de los sistemas periféricos son intervenidas por comunicaciones concéntricas emanadas de una instancia central" (Mascareño, 2003: 7). Esto deriva en dinámicas comunicacionales en las que el sistema político predomina, implicando una injerencia del Estado en las orientaciones adoptadas por otros sistemas.

En la última parte del siglo XX nacen movimientos indígenas en distintas partes del mundo, incluyendo América Latina, con lo cual emerge una semántica del reconocimiento que es progresivamente recogida por las instituciones supranacionales. Surgen en estos años numerosas propuestas de escritura para el mapudungún (véase Zúñiga, 2001), entre las cuales tres destacan por la difusión alcanzada. El primero es el Alfabeto mapuche unificado (1986), creado por lingüistas chilenos y mapuches. Este sistema es ideado con la intención de entregar un estándar para la escritura de la lengua mapuche, en un contexto en el que cada autor empleaba su propio modelo de alfabeto (Gallardo, 1988), teniendo por deseable el logro de una ortografía de transición de fácil acceso para los mapuches alfabetizados en español (Clavería, 2017). El segundo es el Raguileo (1982), diseñado por el lingüista mapuche Anselmo Raguileo, quien persigue un sistema de escritura que remarque la identidad de la lengua mapuche y que exprese de mejor modo sus fonemas (Clavería, 2017; Zúñiga, 2001). Por último, el Azümchefe (1998), confeccionado, tras un proceso participativo, por el Estado chileno, particularmente a través de la Corporación de Desarrollo Indígena, pretende integrar elementos de las propuestas de grafemario más relevantes, ajustándose también a criterios técnicos (Álvarez-Santullano, Forno y Risco, 2015).

Las intenciones de diversas organizaciones y actores por introducir la distinción escribir con $\mathrm{x} /$ no escribir con $\mathrm{x}$ en el entorno de los sistemas de interacción, $\mathrm{y}$ lograr que constituyan para ellos la única alternativa posible de seleccionar, deriva en una serie de conflictos vinculados a lo político, lo identitario y lo técnico. Desde algunos sectores mapuches en que predomina una semántica ligada a la tradición y a la demarcación radical de la diferencia mapuche/no mapuche, el grafemario Raguileo es considerado el único legítimo. Esto debido a que el unificado aparece 
como una imposición de expertos no pertenecientes a la comunidad de habla (Zúñiga, 2001), mientras que el Azümchefe es visto como una apropiación de la lengua por parte del Estado (Álvarez-Santullano, Forno y Risco, 2015; Clavería, 2017). Paralelamente, algunos expertos han emitido juicios críticos respecto del grafemario Raguileo, arguyendo cuestiones vinculadas al modo en que representan gráficamente los fonemas del mapudungún y las dificultades que representa para el tránsito de una alfabetización en español a una en la lengua originaria (Zúñiga, 2001; Clavería, 2017).

En este sentido, la definición de un sistema de escritura para la lengua mapuche está marcada por la disputa por el dominio de la comunicación orientada a introducir en el contexto de los sistemas de interacción la distinción escribir con $\mathrm{x} /$ no escribir con $\mathrm{x}$.

El análisis de estos tres casos aporta evidencia sobre las ventajas que una lectura sistémica de los procesos de planificación lingüística presenta para la comprensión de estos. En efecto, esta aproximación visibiliza que tales iniciativas tienen lugar en el marco de dinámicas comunicacionales conflictuadas, respecto a la regulación ideal del mercado lingüístico. Por ende, será el modo en que se estructure esta comunicación el que determinará qué alternativas aparecen como dotadas de sentido en el entorno de los sistemas de interacción, es decir, para los hablantes insertos en la práctica comunicativa.

\section{CONCLUSIONES}

A lo largo de las últimas décadas se ha planteado la necesidad de contar con una teoría general en el campo de la planificación lingüística. Sin embargo, hasta el presente no se ha logrado satisfactoriamente, por lo que la propuesta planteada en este trabajo intenta ser un aporte en esta dirección. Se ha presentado una aproximación que nace desde una lectura sistémica de los avances teóricos aportados por la sociolingüística y la sociología del lenguaje en la materia. En esta línea, se partió de dos supuestos: (1) que la planificación lingüística constituye un modo de regulación del mercado lingüístico en contextos de contacto entre lenguas -o variantes lingüísticas-, los cuales están marcados por ciertas condiciones sociohistóricas, y (2) que dicho contacto se materializa en los sistemas de interacción, esto es, en la práctica comunicativa de los hablantes.

En este marco, el acercamiento teórico planteado sugiere concebir la planificación lingüística como una regulación sistémica contextual que, ante la imposibilidad de intervenir directamente sobre la operación de los sistemas de interacción -debido a su naturaleza autorreferencial-, busca reorientarla mediante la introducción en el entorno de distinciones que aparezcan como alternativas dotadas de sentido para tales sistemas. 
El análisis de los casos expuestos da cuenta de la aplicabilidad del marco teórico propuesto en el presente trabajo a la realidad empírica. Dicho análisis, además, evidencia que, en contextos de relaciones etnolingüísticas asimétricas, el conflicto permite la emergencia y generalización de semánticas que propician nuevas formas de regulación de los mercados. Desde aquellas el capital lingüístico minoritario aparece como una alternativa dotada de sentido en el entorno de los sistemas de interacción. Simultáneamente, la revisión de estos casos visibiliza la relevancia del sistema jurídico en los procesos de planificación, en cuanto la legislación aparece como un mecanismo fundamental de cristalización de nuevas formas de comunicación.

No obstante lo anterior, al aplicar este enfoque a la realidad latinoamericana es preciso reconocer que estas sociedades no se ajustan a las características de las sociedades modernas sobre las que teorizaba Luhmann. Por lo tanto, se vuelve necesario tratarlas, junto con Mascareño (2003), como sociedades diferenciadas concéntricamente, en las cuales el Estado adquiere el rol de instancia central, siendo el espacio desde donde se configuraron y desarrollaron las sociedades del subcontinente. Esto hace que sus distinciones sean de fundamental relevancia para la operación de otros sistemas. En este sentido, en el caso de América Latina, en general, y de Chile, en particular, el cómo se equiparan las relaciones sociolingüísticas entre la lengua oficial y las lenguas indígenas depende en gran medida de las distinciones trazadas por el Estado.

\section{REFERENCIAS}

Aikio-Puoskari, U. (2018). Revitalization of Sámi languages in three nordic countries: Finland, Norway, and Sweden. En Hinton, L.; Huss, L. y Roche, G. (Eds.). The Routledge handbook of language revitalization (pp. 355-363). Londres: Routledge.

Álvarez-Santullano, P.; Forno, A. y Risco, E. (2015). Propuestas de grafemarios para la lengua mapuche: desde los fonemas a las representaciones políticoidentitarias. Alpha, 40, 113-130.

Amorós-Negre, C. (2017). Different paradigms in the history of Spanish language policy and planning. Journal of Multilingual and Multicultural Development, 38(1): 65-78.

Barber, K. (2008). 'Indigenous rights' or 'racial privileges': the rethoric of 'race' in New Zeland politics. The Asia Pacific Journal of Anthropology, 9(2), 141-156.

Beach, H. (1994). The Saami of Lapland. En Minority Rights Group (Ed.). Polar peoples. Self-determination \& development (pp. 147-205). London: Minority Rights Group.

Bourdieu, P. (1991). Language and symbolic power. Cambridge: Polity Press. 
Bourdieu, P. (1999). El mercado lingüístico. En Cuestiones de sociología (pp. 120136). Madrid: Istmo.

Bull, T. (2012). Against the mainstream: universities with an alternative language policy. International Journal of the Sociology of Language, 216: 55-73.

Clavería, A. (2017). Alfabetos para escribir y para luchar. Consideraciones sociopolíticas en torno a la falta de estandarización de la escritura en mapudungun. Cultura, Hombre, Sociedad, 27(2): 215-228.

Cooper, R. (1997). La planificación lingüistica y el cambio social, Madrid: Cambridge University Press.

Crow, J. (2015). Mapudungun and the contested process of (nation) state building. En Langer, N. y Havinga, A. (Eds.). Invisible languages in the nineteenth century (pp 51-70). Oxford: Peter Lang.

Deumert, A. (2009). Language planning and policy. En Mesthrie, R.; Swann, J.; Deumert, A. y Leap, W. (Eds.). Introducing sociolinguistics (pp. 371-406). Edinburgh: Edinburgh University Press.

Diamond, B. (2007). "Allowing the listener to fly as they want to": Sami perspectives on indigenous CD production in Northern Europe. The World of Music, 49(1): 23-48.

Durán, T. y Ramos, N. (1987). Incorporación del español por los mapuches del centro-sur de Chile durante el siglo XIX. Lenguas Modernas, 14: 179-196.

Ethnologue (2018a). Saami, North. Disponible en: https:/www.ethnologue.com/ language/sme

Ethnologue (2018b). Maori. Disponible en: https:/www.ethnologue.com/language/mri

Falch, T.; Selle, P. y Strømsnes, K. (2016). The Sámi: 25 years of indigenous authority in Norway. Ethnopolitics, 15(1): 125-143.

Fishman, J. (1995). Sociología del lenguaje (4ta Ed.). Madrid: Cátedra.

Fishman, J. (2001). 'Why Is it so Hard to Save a Threatened Language?', en J. Fishman (Ed.). (2001). Can Threatened Languages Be Saved? Clevedon: Multilingual Matters, 1-22.

Gallardo, A. (1988). El desarrollo de la escritura en lenguas vernáculas de Chile. En Sociedad Chilena de Lingüística (Ed.). Alfabeto Mapuche Unificado (pp. 37-60). Temuco: Universidad Católica de Temuco.

Gaski, H. (2011). Song, poetry and images in writing: Sami literature. Nordlit, 27: 33-54.

Goverment of Norway (2007). Act no. 56 concerning the Sameting (the Sami parliament) and other Sami legal matters (the Sami Act). Disponible en: https:// www.regjeringen.no/en/dokumenter/the-sami-act-/id449701/

Goverment of Norway (2014). Act no. 61 relating to primary and secondary education and training. Disponible en: https:/www.regjeringen.no/contentassets/ b3b9e92cce6742c39581b661a019e504/education-act-norway-with-amend- 
ments-entered-2014-2.pdf

Grenoble, L. y Whaley, L. (2005). Saving languages. An introduction to language revitalization. Cambridge: Cambridge University Press.

Gupta, A. (1995). Blurred boundaries: the discourse of corruption, the culture of politics, and the imagined state. American Ethnologist, 22(2): 375-402.

Heller, M. (2013). Crosswords: Language, education and ethnicity in French Ontario, Berlín: Mouton de Gruyter.

Higgins, R. y Rewi, P. (2015). ZePA - Right-shifting: Reorientation towards normalisation. En Higgins, R.; Rewi, P. y Olsen-Reeder, V. (Eds.), The Value of the Maori Language. Wellington: Huia.

Hornberger, N. (2006). Frameworks and models in language policy and planning. En Ricento, T. (Ed). An Introduction to Language Policy: Theory and Method (pp. 24-41). Malden: Blackwell Publishing.

Hornberger, N. (2008). Introduction: Can schools save indigenous languages? Policy and practice on four continents. En Can schools save indigenous languages? Policy and practice on four continents (pp. 1-12). Nueva York: Palgrave Macmillan.

Joseph, B. (2015). Language contact. En Wright, J. (Ed.). International encyclopedia of the social and behavioral sciences (2da Ed.) (Vol. 13) (pp. 300-306). Ámsterdam: Elsevier.

König, J. (2005). Discursos de identidad, Estado-nación y ciudadanía en América Latina. Historia y Sociedad, 11: 9-31.

Lagos, C. y Espinoza, M. (2013). Planificación lingüística de la lengua mapuche en Chile a través de la historia. Lenguas Modernas, 42(2): 47-66.

Liddicoat, A. y Baldauf, R. (2008). Language planning in local contexts: agents, contexts and interactions. En Liddicoat, A. y Baldauf, R. (Eds.). Language policy and planning. Language planning in local contexts (pp. 3-17). Clevedon: Multilingual Matters.

Luhmann, N. (1996). Introducción a la teoría de sistemas. México D.F.: ITESO/ Universidad Iberoamericana.

Luhmann, N. (1998). Complejidad y modernidad. De la unidad a la diferencia. Madrid: Trotta.

Luhmann, N. (2006). La sociedad de la sociedad. México D.F.: Herder/Universidad Iberoamericana.

Mahrooqi, R. y Asante, C. (2012). Revitalizing the Maori language: a focus on educational reform. Social Sciences \& Humanities, 20(4): 1035-1048.

Markelin, L., y Husband, C. (2013). Contemporary dynamics in Sámi media in the Nordic States. Media International Australia, 149(1): 70-81.

Mascareño, A. (2003). Teoría de sistemas en América Latina. Conceptos fundamentales para la descripción de una diferenciación funcional concéntrica. Persona y Sociedad, 17(2), 1-20. 
Mascareño, A. (2011). Sociología de la intervención: orientación sistémica contextual. Revista MAD, 25: 1-33.

May, S. (1998). Language and education rights of indigenous peoples. Language, Culture and Curriculum, 11(3), 272-296.

May, S. (2015). Language policy and political theory. En Hult, F. y Johnson, D. (Eds.). Research methods in language policy and planning. A practical guide (pp. 45-55). Sussex Occidental: Wiley Blackwell.

May, S. y Hill, R. (2018). Language revitalization in Aorearoa/New Zealand. En Hinton, L.; Huss, L. y Roche, G. (Eds.). The Routledge handbook of language revitalization (pp. 309-319). Londres: Routledge.

Minde, H. (2005). Assimilation of the Sami - Implementation and consequences. Journal of Indigenous Peoples Rights, 3: 6-33.

Nahir, M. (1998) Micro language planning and the revival of Hebrew: A schematic framework. Language in Society, 27: 335-357.

Nugent, D. (2004). Governing states. En Nugent, D. y Vincent, J. (Eds). A companion to the anthropology of politics (pp. 198-215). Malden: Blackwell Publishing.

Omdal, H. (2006). Language Planning: Standardization. En Ammon, U.; Dittmar, N.; Mattheier, K. y Trudgill, P. (Eds). Sociolinguistics. An international handbook of the science of language and society (pp. 2384-2394). Berlín/Nueva York: Walter de Gruyter.

Pietikäinen, S. (2008). Broadcasting indigenous voices. Sami minority media production. European Journal of Communication, 23(2): 173-191.

Pinto, J. (2003). La formación del estado y la nación, y el pueblo mapuche: de la inclusión a la exclusión. Santiago: DIBAM.

Reedy, T. (2000). Te reo māori: the past 20 years and looking forward. Oceanic Linguistics, 39(1): 157-169.

Ricento, T. (2006). Language policy: theory and practice - An introduction. En An introduction to language policy. Theory and method (pp. 10-23). Blackwell Publishing: Oxford.

Rojas, D. (2013). Actitudes e ideologías de hispanohablantes en torno a las lenguas indígenas en el Chile del siglo XIX. Lenguas Modernas, 42(2), 85-98.

Romaine, S. (2002). The impact of language policy on endangered languages. International Journal on Multicultural Societies, 4(2): 194-212.

Stortinget, (2014). The Constitution. Disponible en: https://www.stortinget.no/ en/ Grunnlovsjubileet/In-English/The-Constitution---Complete-text/

Todal, J. (2003). The Sámi school system in Norway and international cooperation. Comparative Education, 39(2): 185-192.

Vallejos, A. (2008). Conflictos socioambientales en la periferia de la modernidad. Apuntes para la intervención en contextos locales. Revista de Ciencias Sociales, 14(2): 240-254. 
Wiley, T. (1996). Language planning and policy. En McKay, S. y Hornberger, N. (Eds.), Sociolinguistics and language teaching (pp. 103-47). New York: Cambridge University Press.

Wilke, H. (2006). La transformación de la democracia como modelo de orientación de las sociedades complejas. Estudios Públicos, 102: 179-201.

Wright, S. (2004). Language policy and language planning. From nationalism to globalisation. Nueva York: Palgrave McMillan.

Zúñiga, F. (2001). Escribir en mapudungun. Una nueva propuesta. Onomázein, 6, 263-279. 